- Develop closer working relationships with the Speech and Language, Dietician and Head and Neck services.

Approach Used Four cornerstones of care used:-

- Skin care: application of regular skin cream to hydrate skin, prevent infection and encourage self-management

- Manual Lymphatic Drainage (MLD): specialised gentle massage to aid and stimulate drainage.

- Simple Lymphatic Drainage; patient learns to move the build-up of fluid

- Exercises (facial):- Encourages muscle movement, aids movement of lymphatic fluid. Breathing exercises stimulate deeper lymphatic vessels in chest and abdomen to enhance lymph drainage.

- Compression worn to maintain shape, reduce swelling.

New treatment modalities (accompanied by modified MLD):

- Low level laser therapy: infra-red wavelength penetrates deep into tissues, aids softening of fibrosis and scar tissue, useful in treating cording.

- Deep oscillation therapy: - penetrates deep into body tissue triggering lasting resonance

- Kinesio Tape: increases body's ability to drain lymphatic drainage to healthy lymph nodes

These innovative treatments have proved to be an effective adjunct to our existing specialist knowledge and enhanced treatments offered to patients

Measurements pre and post treatment compared using photographs

Patients complete questionnaire after each session, record any immediate changes to symptoms.

Outcomes Increased referrals for treatment to head, neck and face

$2011 / 2012$ - 5 referrals

2012/2013 - 17 referrals

Development of a closer working relationship with the surgical head and neck multidisciplinary team at hospital.

Patient comments I don't feel that everyone is looking at my face as they did before, I felt like I was the elephant man, much happier and I went for a walk with the wife, I wouldn't have done that a few weeks ago.

I was able to eat normal food after treatment for that day

\section{P161 MEANING OF PAIN FOR PATIENTS WITH ADVANCED CANCER AND HOW IT INFLUENCES BEHAVIOUR: A QUALITATIVE RESEARCH STUDY}

Rebecca Jennings. St Joseph's Hospice, London, United Kingdom

10.1136/bmjspcare-2013-000591.183

Background Health related quality of life incorporates physical function and independence which are high priorities for patients with advanced cancer. These two inter-related domains can be compromised through the experience of cancer-related pain. Patient-held meanings of cancer pain may have potential to influence behaviours which determine functionality and quality of life.

Study aim To explore the meanings of pain among patients living with advanced cancer and to understand how these influence functional behaviours, with the aim of developing clinical recommendations to inform patient care.

Design Participants underwent in-depth, semi-structured, qualitative interviews. Data was analysed using Interpretative Phenomenological Analysis to identify emergent themes.
Setting/Participants: Ten patients with advanced stage cancer experiencing cancer-related pain were recruited from an InnerLondon hospice palliative care service.

Results Meaning of pain in advanced cancer is inextricably linked with loss and bereavement. Meaning of pain is a dynamic, temporal process underpinned by the following themes: (i) nature of cancer-related pain, (ii) multidimensional impact of pain on patients' function and behaviour, (iii) 'Web of Loss': a complex network of losses that perpetuates cycles of further loss and (iv) hope and appreciation of life. The data suggests meaning of pain has an indirect influence on behaviour mediated by the coping strategies patients adopt. Meaning of pain has potential to promote both constructive and maladaptive responses in functional behaviour.

Conclusions Findings further our understanding of the experience of cancer-related pain and inform bio-behavioural approaches to pain management in palliative care. Recommendations include (i) tailoring pain management to dedicate greater attention to the behavioural dimension of cancer-related pain (ii) timely identification and management of preparatory grief in patients with advanced cancer and (iii) promotion of constructive coping strategies to support patients to make sense of their pain and maintain functionality within the limitations of advancing disease.

\section{P162 LOST IN TRANSLATION; PHYSIOTHERAPISTS' ATTITUDES AND BELIEFS TOWARDS PALLIATIVE CANCER PATIENTS IN THE ACUTE HOSPITAL SETTING}

Helen Hutchinson. Princess Alice Hospice, Esher, UK

\subsection{6/bmjspcare-2013-000591.184}

Abstract Aim The study aimed to elucidate any attitudes acute hospital physiotherapists may have towards palliative cancer patients.

Background With advances in medical technology, increasing numbers of patients are 'living with' a terminal cancer diagnosis for longer periods; this coupled with changes in government policy, is resulting in patients being treated closer to home rather than in specialist centres, bringing physiotherapists into increasing contact with this patient group.

Methods Semi-structured interviews (analysed utilising Colaizzi's method of analysis), of the first twelve physiotherapists from an acute hospital in the UK who volunteered for the study and who met the inclusion criteria.

Results Two clusters of themes were identified, namely Attitudes and Issues. There were four sub-divisions of Attitudes; terminology, physiotherapists' perceptions, other professionals' perceived attitudes towards physiotherapists, and patients and their families perceived attitudes towards physiotherapists' role with terminally ill cancer patients.

"I'd say there are a lot of physio's that think it's probably a little bit pointless"

"try and maintain their quality of life ... by their thinking... and hopefully try and meet it."

There were six sub-divisions of issues: communication, education, emotions, boundaries, referring onward and time.

"I think they're [physiotherapists] scared that that's going to be asked of you...will I walk again?"

Conclusions Some physiotherapists understand and apply their skills to improve the quality of life of palliative cancer patients. However it is suggested that the majority of physiotherapists regard these patients as 'dying from' rather than 'living with' a 